\title{
PREMIERS RESULTATS SUR LES COMPORTEMENTS DE SUBSISTANCE SOLUTRÉENS À LA GROTTE ROCHEFORT (MAYENNE, FRANCE)
}

\author{
First results from Solutrean subsistance behaviour from \\ Rochefort Cave (Mayenne, France)
}

\author{
Céline Bemilli ${ }^{1}$ y Stéphan Hinguant ${ }^{2}$
}

Recibido el 3 de abril de 2013. Aceptado el 17 de julio de 2013

Résumé. Le site de la grotte Rochefort (Mayenne, France) a livré depuis 2005 plusieurs centaines de restes fauniques issus des couches solutréennes. Le renne et le cheval en sont les principales composantes et reflètent le fond commun du régime alimentaire des solutréens. Nous présenterons ici les premiers résultats de l'analyse archéozoologique concernant l'exploitation de ces deux principaux taxons. Plusieurs recollages ont été réalisés. L'exceptionnelle conservation des restes permet, outre une excellente lecture des traces de découpe, une première reconstitution des modalités d'acquisition et d'exploitation des animaux, ainsi que leur saison d'abattage. La rareté des ensembles fauniques solutréens fouillés et étudiés récemment donne à celui de la grotte Rochefort un impact particulier et inédit.

Mots clés: Solutréen, subsistance, renne, cheval, grotte Rochefort, Mayenne.

Abstract. Several hundred faunal remains were discovered since 2005 in the Solutrean's level at Rochefort cave (Mayenne, France). Reindeer and Horse are the main taxa identified and are the baseline of the Solutrean diet. We present here the preliminary results of the faunal analysis and discuss the exploitation of these two main taxa. A number of refits were possible. At the same time, the exceptional preservation of the artefacts together with well-defined cut marks allows to reconstruct procurement and use patterns of these animals as well as the season they were killed. Recently excavated and analysed Solutrean faunal assemblages are rare which make the yet unreported Rochefort cave faunal assemblage even more important and unprecedented.

Keywords: Solutrean, subsistance, reindeer, horse, Rochefort cave, Mayenne.

\section{LA GROTTE ROCHEFORT}

La grotte Rochefort, sur la commune de Saint-Pierresur-Erve (Mayenne, France), est une cavité appartenant à l'ensemble des grottes dites du canyon de Saulges, toutes localisées sur les deux rives de l'Erve sur environ 1,5 km de son parcours (Fig. 1). Son porche d'entrée, haut et étroit, s'ouvre en milieu de falaise à environ $15 \mathrm{~m}$ au dessus du lit actuel de la rivière. Fréquentée depuis le Paléolithique, elle est une des cavités les plus vastes du site, dont le développement, composé d'un réseau supérieur et un réseau inférieur, atteint $250 \mathrm{~m}$. Seul le premier a été accessible au cours des temps préhistoriques, comprenant un couloir d'accès long de $24 \mathrm{~m}$ et une grande salle dans laquelle il débouche. Malgré

(1) INRAP. Centre archéologique de Grand Quevilly. 30 boulevard de Verdun. Immeuble Jean Mermoz. 76120 Grand-Quevilly (France). UMR 7209. Archéozoologie, Archéobotanique: sociétés, pratiques et environnements. Muséum national d'histoire naturelle. Paris (France). celine.bemilli@inrap.fr

(2) INRAP. Base archéologique de Cesson-Sévigné. 37 rue du Bignon. 35577 Cesson-Sévigné (France). CNRS. UMR 6566 «CReAAH». Universités de Rennes 1 et 2. Ministère de la Culture. Rennes (France). stephan.hinguant@inrap.fr 
plusieurs fouilles à la fin du XIXe siècle et dans les années 1930, qui ont permis d'identifier une présence paléolithique dans la grotte, les surfaces disponibles pour des recherches archéologiques modernes demeuraient importantes. La cavité a donc fait l'objet d'une reprise des recherches en 2001 sous la responsabilité de l'un d'entre nous (S.H) et plusieurs niveaux d'occupations très peu perturbés par les fouilles anciennes ont ainsi pu être mis au jour, dont une remarquable couche solutréenne (Hinguant et Colleter 2010; Hinguant et Biard 2013).

Les fouilles actuelles montrent clairement que nous nous trouvons en périphérie de l'occupation solutréenne, dans un secteur de la salle plus étroit, moins haut et que n'atteint pas le faible éclairage naturel encore perceptible à l'extrémité du couloir. Les rejets semblent importants et le net gradient de densité suggère bien que le cœur de I'habitat se trouve dans la partie la plus vaste et la mieux éclairée de la salle (Fig. 2). Pour autant, si aucune organisation spatiale n'est identifiée, la présence d'un rejet de foyer et d'une structure aménagée avec des blocs de calcaire, en limite d'emprise de fouille, soulignent néanmoins l'existence d'au moins un sol d'occupation. Les raccords et remontages entre pièces lithiques ou osseuses montrent par ailleurs que des zones préférentielles d'activités existent. Malgré d'évidents problèmes post-dépositionnels, notamment liés à la cryoturbation et aux ruissellements, la lecture spatiale apporte donc de réelles informations, opportunément renforcées par la remarquable qualité de conservation des objets mobiliers.

Stratigraphie et datations radiocarbone placent l'occupation au cœur du Solutréen, entre 19000 et 20000 BP, chronologie confirmée par la culture matérielle ne comptant ni pointes à face plane ni pointes à cran mais où domine la feuille de laurier (Fig. 3). Il faut souligner ici la remarquable homogénéité de cette unité solutréenne, excluant tout mélange possible avec des chronocultures antérieures et/ ou postérieures. L'unité solutréenne de la grotte, à ce stade des recherches, est d'une puissance maximale de 50 à 60 $\mathrm{cm}$, composée de clastes calcaires issus de l'érosion d'une cheminée de soutirage et d'une matrice sablo-argileuse comblant les interstices. Six subdivisions sont enregistrées (4.1 à 4.6), correspondant à des changements de structures, de textures et de couleur dans la stratigraphie, mais aussi à des densités différentes de mobilier. Si quelques mélanges verticaux sont notés, I'homogénéité de chacune des strates semble préservée, notamment pour les subdivisions 4.2 et 4.3 , sans que l'on sache néanmoins si elles correspondent à la superposition d'occupations successives (saisonnières ou autres) ou à un palimpseste.

Les différentes campagnes de fouilles de la grotte Rochefort ont permis de découvrir un matériel archéologique variés et en très bon état de conservation. Outre une série lithique exceptionnelle de près de 2000 artefacts, plus de
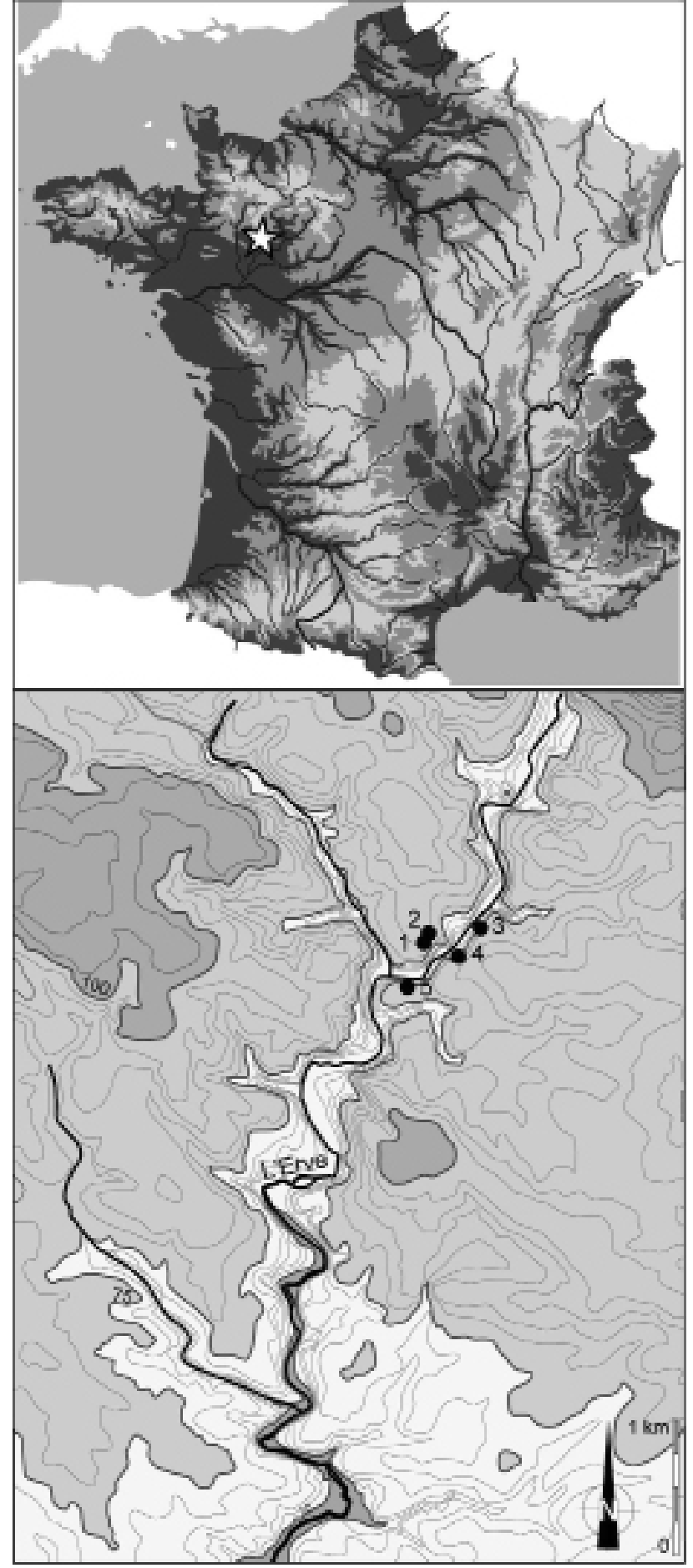

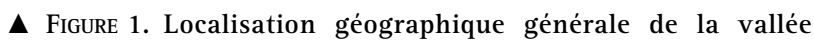
de l'Erve et des principales grottes du canyon de Saulges : 1 , grotte Rochefort ; 2 , grotte de la Chèvre ; 3, le Plessis ; 4, grotte Margot ; 5, la Dérouine/ Mayenne-Sciences ( $\odot$ Rozenn Colleter, Inrap).

5500 restes de faune ont été enregistrés. Bien que la grande faune mammalienne soit la mieux documentée, des restes de microfaune et de poisson ont également été découverts. $A$ côté de ces vestiges liés aux activités de taille et de subsistance, le site a également livré une industrie en os, des éléments de parures, des plaquettes gravées, ainsi que quelques 


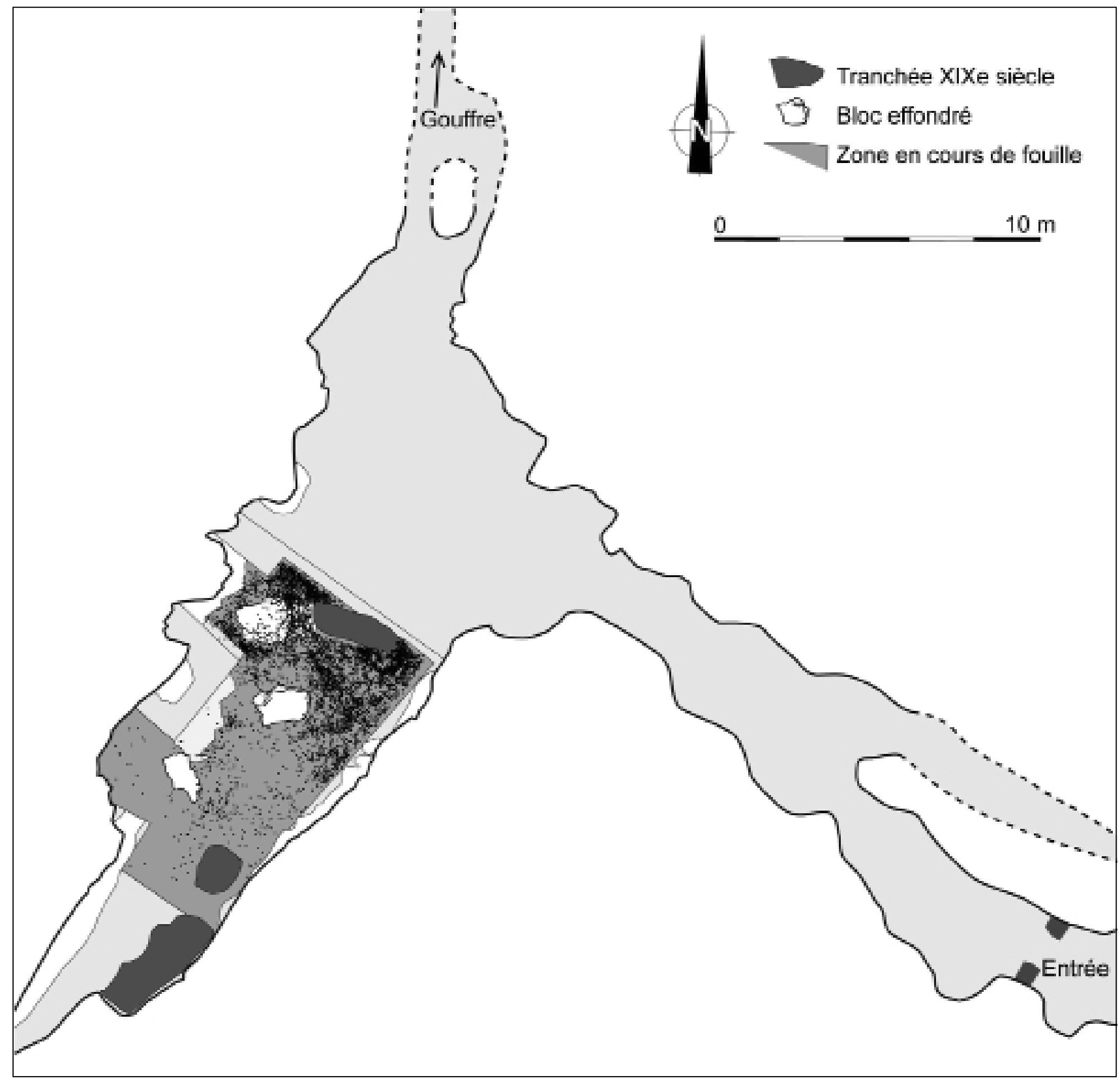

\ Figure 2. Plan de la grotte Rochefort et répartition spatiale du mobilier Solutréen ( ${ }^{\circ}$ Rozenn Colleter, Inrap).

pièces inédites comme des côtes gravées et un fragment d'ivoire de mammouth incisé (Hinguant et al. 2012).

A ce stade des recherches, toutes les données convergent donc pour faire de cette occupation solutréenne en grotte profonde un véritable habitat, le seul à ce jour connu pour ce techno-complexe au nord de la Loire.

\section{LA FAUNE SOLUTRÉENNE}

\subsection{Les effectifs}

Environ 5500 restes osseux fauniques ont été récoltés durant les cinq années de fouille. Ils présentent un état de conservation exceptionnel même si la fragmentation des os est très importante, ce qui explique le taux de détermination de $18 \%$ avec 906 restes déterminés (Tab. 1). On peut y ajouter une soixantaine de restes de lagomorphes, d'oiseaux et de poissons ainsi que plus de 7000 restes de microfaune (Hinguant et Colleter 2010).

Une vingtaine d'espèce est identifiée mais ce sont le renne (Rangifer tarandus) et le cheval (Equus ferus) qui constituent la grande majorité des vestiges, regroupant 84,2 $\%$ du NRDt avec respectivement 475 et 306 restes (Tab. 1). Ce sont les données issues de l'analyse de ces deux espèces qui seront essentiellement présentées ici, les analyses archéozoologiques et taphonomiques des restes indéterminés n'étant à ce jour pas encore achevées. 


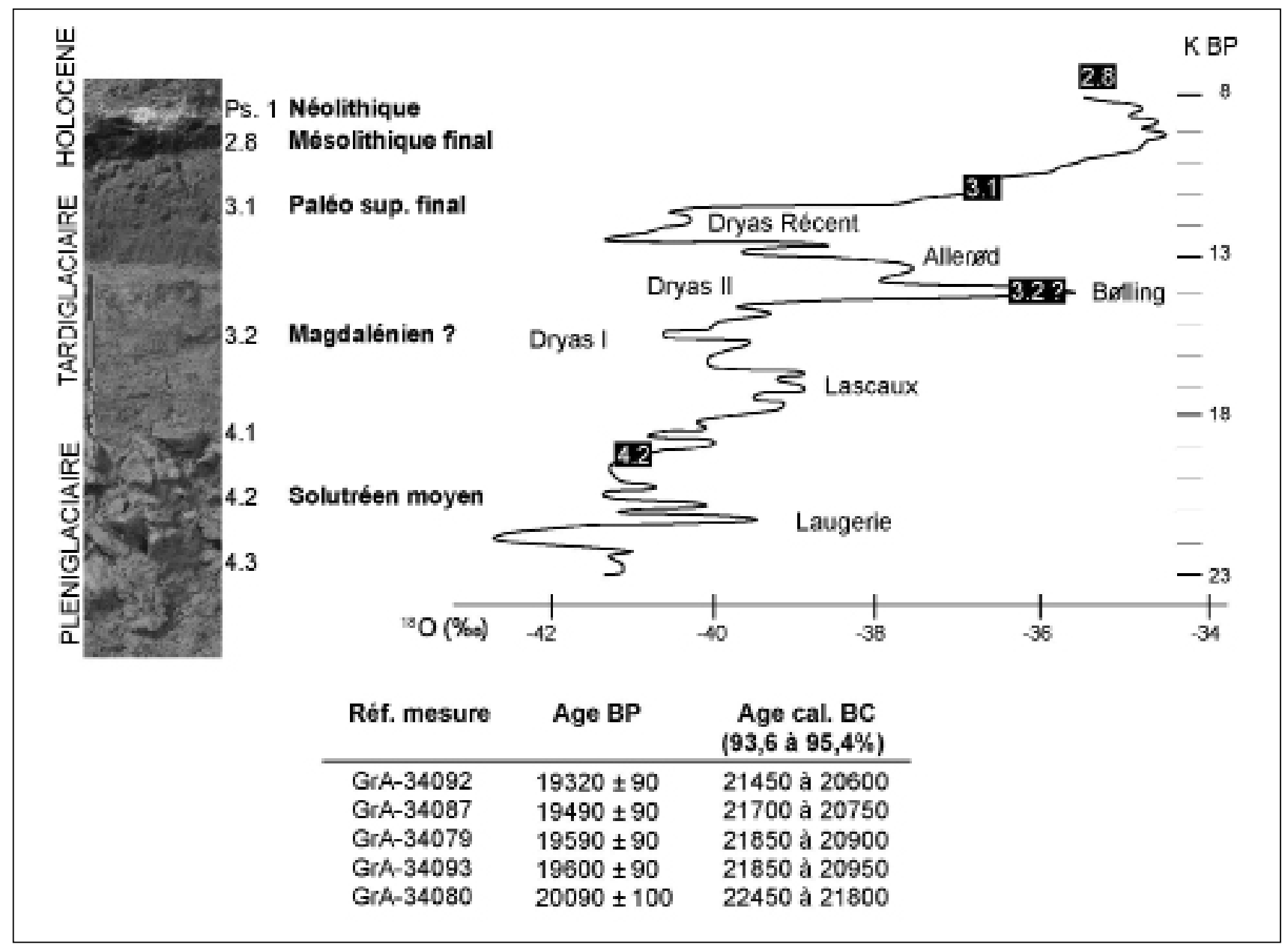

A Figure 3. Stratigraphie du remplissage supérieur de la grotte Rochefort et datations 14C des niveaux solutréens ( ${ }^{\circ}$ Rozenn Colleter et Stéphan Hinguant, Inrap).

Exceptés l'ours brun (Ursus arctos 42 restes), le bouquetin des Alpes (Capra Ibex, 24 restes), le loup (Canis lupus, 15 restes) et les renards roux (Vulpes vulpes, 7 restes) et polaire (Alopex lagopus, 5 restes), les autres taxons sont documentés par moins de 5 restes. II s'agit du chevreuil (Capreolus capreolus, 4 restes), du sanglier (Sus scrofa scrofa, 3 restes), du rhinocéros laineux (Coelodonta antiquitatis, 2 restes), du mammouth (Mammuthus primigenius, 3 restes), de la panthère des neiges (Panthera pardus, 1 reste), du chat sauvage (Felis silvestris, 2 restes), d'un mustélidé (Mustela sp., 1 reste) auxquels il faut ajouter 7 restes d'un grand cervidé indéterminé, 4 restes de renard indéterminé, 2 restes d'un petit carnivore et deux restes de boviné indéterminé (Bison/ Bos). Les effectifs dénombrés à ce jour pour chaque espèce font état de 15 rennes, 8 chevaux, 2 ours, 3 renards roux et 2 renards polaires, tous les autres taxons n'étant documentés que par un seul individu (Tab. 1).

Des traces de découpe ont été relevées sur les restes de renne, de cheval, de bouquetin, d'ours et de loup certifiant la consommation des trois premiers et au moins la récupération de la peau des deux derniers par les chasseurs solutréens.
D'un point de vue écologique et paléoenvironnemental, ce cortège faunique reflète un environnement découvert de climat froid. Mis en relation avec les datations radiocarbone et certains taxons de microfaune, il peut être contemporain du maximum de froid du stade isotopique 2. Deux espèces très caractéristiques ne sont pourtant pas encore déterminées à la grotte Rochefort ni d'une manière générale dans la vallée de l'Erve. II s'agit du bœuf musqué Ovibos moschatus et de l'antilope saïga Saiga tartarica, espèces par exemple présentes dans le Solutréen de Laugerie-Haute Ouest aux Eyzies-de-Tayac (Dordogne) (Madelaine 1989; Castel et Madelaine 2006; Delpech 2012). En revanche, quelques espèces suggèrent la présence de couverts forestiers plus ou moins importants dans l'environnement des grottes. L'ours, pour lequel les restes déterminés à l'espèce correspondent tous à l'ours brun, affectionne plutôt les milieux arborés mais sa présence en milieu ouvert n'est pas aberrante. II en est de même pour le Sanglier. Par contre, lynx et chat sylvestre sont des carnivores de massifs forestiers denses qui n'ont cependant pas forcément besoin d'un vaste territoire si se trouvent suffisamment de proies disponibles à leur portée. Dans le contexte périglaciaire envisagé pour la 


\begin{tabular}{|l|l|r|r|r|}
\hline & & NR & $\%$ NR & NMI \\
\hline Artiodactyles & & & & \\
\hline Boviné & Bos/Bison & 2 & 0,2 & 1 \\
\hline $\begin{array}{l}\text { Bouquetin des } \\
\text { Alpes }\end{array}$ & Capra ibex & 24 & 2,6 & 1 \\
\hline Chevreuil & Capreolus capreolus & 4 & 0,4 & 1 \\
\hline Renne & Rangifer tarandus & 475 & 52,4 & 15 \\
\hline Grand cervidé & Cervus elaphus? & 7 & 0,8 & 1 \\
\hline Sanglier & Sus scrofa scrofa & 3 & 0,3 & 1 \\
\hline Périssodactyles & & & & \\
\hline Cheval & Equus ferus & 306 & 33,8 & 8 \\
\hline $\begin{array}{l}\text { Rhinocéros } \\
\text { laineux }\end{array}$ & $\begin{array}{l}\text { Coelodonta } \\
\text { antiquitatis }\end{array}$ & 2 & 0,2 & 1 \\
\hline Proboscidien & & & & \\
\hline Mammouth & $\begin{array}{l}\text { Mammuthus } \\
\text { primigenius }\end{array}$ & 3 & 0,3 & 1 \\
\hline Carnivores & & & & \\
\hline Ours brun & Ursus arctos & 42 & 4,6 & 2 \\
\hline Loup & Canis lupus & 15 & 1,7 & 1 \\
\hline Renard roux & Vulpes vulpes & 7 & 0,8 & 3 \\
\hline Renard polaire & Alopex lagopus & 5 & 0,6 & 2 \\
\hline $\begin{array}{l}\text { Renard } \\
\text { indéterminé }\end{array}$ & Alopex/Vulpes & 4 & 0,4 & \\
\hline Panthère & Panthera pardus & 1 & 0,1 & 1 \\
\hline Lynx & Lynx sp. & 2 & 0,1 & 1 \\
\hline Chat sauvage & Felis silvestris & 0,1 & 1 \\
\hline Mustelidé & Mustela & & \\
\hline Petit carnivore & & & \\
\hline
\end{tabular}

A Tableau 1. Composition de la faune de la grotte Rochefort (NR, nombre de reste ; NMI, nombre minimum d'individu).

région, l'environnement rocailleux de la vallée de l'Erve et les bosquets forestiers périphériques devaient parfaitement leur convenir. Si des intrusions de petits fossiles provenant des couches supérieures peuvent en partie expliquer la présence de certains de ces restes " tempérés ॥ dans la couche solutréenne, d'autres sont clairement associés à ce niveau et un très grand nombre de fossiles de microfaune corrobore bien cette coexistence de milieux ouverts et arides (Microtus gregalis, Dicrostonyx torquatus, Spermophilus citellus...) et d'espaces plus humides voire boisés (Microtus agrestis, Arvicola...). En limite septentrionale de la toundra arborée, la vallée de l'Erve et ses environs devaient voir se développer des biotopes favorables à des espèces aux exigences si différentes.

Une autre particularité de l'assemblage faunique de la grotte Rochefort réside en la présence du bouquetin des Alpes sous une latitude aussi septentrionale. En regard de la topographie régionale, où les zones d'escarpements rocheux indispensables à cette espèce sont plutôt rares et de faible extension, ce constat reste à expliquer.

Enfin, la part occupée par les carnivores n'est pas négligeable avec 4,7\% mais il faut souligner l'absence totale de I'hyène, ce qui n'est pas sans conséquences sur la qualité et donc l'interprétation de l'assemblage osseux.

\subsection{Méthodologie}

Les restes osseux ont été décomptés en nombre de restes (NR, Poplin 1976 a et b) qui correspond au NSIP anglo-saxon (Grayson 1984) avec la subdivision NRDt pour les restes déterminés totalement. Les nombres minimum d'individus (NMI, Poplin, ibid) ont été calculés soit selon la fréquence (NMIf) la plus forte de chaque partie squelettique (NMPS) en tenant compte de la latéralisation. Ce NMPS se rapproche du MAU de Binford (Binford 1981). Des nombres minimums d'individus de combinaison (NMIc) ont pu être obtenus en combinant les données sur les âges dentaires et épiphysaires (Poplin 1976 b et c). Nous avons choisi, pour illustrer les taux de survie, le \% PO de Grigson et Mellars (1987) '.

Les données concernant la saisonnalité ont été obtenues grâce à la présence d'individus juvéniles et reposent sur l'estimation de l'âge des animaux au moment de leur mort. Nous avons utilisé les référentiels de Cornevin et Lesbre (1894) et Guadelli (1998) ainsi que la collection d'incisives du Muséum national d'Histoire naturelle de Paris pour le cheval et ceux de Bouchud (1966) et de Spiess (1979) pour le renne.

\section{ANALYSE TAPHONOMIQUE}

La conservation des restes de faune dans les niveaux solutréens de la grotte Rochefort est excellente et le tamisage systématique des sédiments a permis la récolte des plus petits éléments comme la microfaune.

Les principales altérations observables sont la présence de voiles de calcite et de concrétion sur certains restes. L'action des carnivores est restreinte et se traduit par des traces de mâchouillage et de crocs (pits) - dont la taille exclue le renard et l'hyène ( $N R=7$ pour le cheval et $N R=8$ pour le renne). Parallèlement, une dizaine de petits os (phalanges, carpiens et tarsiens) présentent des traces de digestions.

L'excellente conservation des os se traduit par la présence de toutes les parties squelettiques, même les plus fragiles, comme les cartilages intercostaux.

La morphologie des fractures évoque une fracturation sur os frais, principalement d'origine anthropique comme

(') II s'agit d'un pourcentage de survie qui se calcule, pour chaque partie anatomique, en considérant le NMPS total (NMPS droite + NMPS gauche + NMPS non latéralisé) divisé par le NMPS attendu pour le NMI le plus élevé de l'espèce, le tout multiplié par cent. Dans le cas où un NMlc a pu être réalisé, c'est sur la base de celui-ci que sera calculé le pourcentage de survie. 
l'atteste la présence d'esquilles de percussion. Aucun os long entier ni de diaphyses sous forme de cylindre n'a été enregistrée et des épiphyses entières sont présentes.

Ces différents facteurs croisés permettent d'écarter les carnivores comme agents principaux d'accumulation de I'assemblage osseux solutréen de la grotte Rochefort. Leur intervention postérieure à l'occupation humaine est toutefois attestée et on ne peut exclure leur responsabilité dans la présence de certaines espèces de petite taille.

Le bon état des surfaces a permis une très bonne lecture des traces de découpe qui sont pourtant peu abondantes (4,6\% pour le renne et 4,5\% pour le cheval).

Enfin, le nombre de restes brûlés est assez faible et correspond à une combustion de faible intensité $(7,6 \%$ pour l'ensemble; 2,1\% pour le renne et $2 \%$ cheval).

\section{ARCHÉOZOOLOGIE DES DEUX PRINCIPAUX TAXONS}

\subsection{La forte présence du renne}

Le renne est l'espèce la mieux représentée avec 375 restes (Tab. 2), soit 52,4\% du NRDt (nombre de reste déterminé totalement) et un NMIc (nombre minimum d'individu de combinaison) de 15 individus (calculé à partir des stades d'éruption et d'usure dentaire selon la méthode de Hufthammer 1995).

Toutes les régions anatomiques (NMPS) sont documentées avec une surreprésentation des métapodes (60\% $\% \mathrm{PO}^{2}$ pour les métacarpes et $50 \%$ PO pour les métatarses, Fig. 4). Les autres parties squelettiques les mieux documentées sont I'humérus (26,6\% P0), suivi des autres os longs représentés dans des valeurs comprises entre 13 et $20 \%$ PO.

Même les plus petits os (carpes) sont présents, en revanche, plusieurs segments du rachis manquent: axis, vertèbres lombaires, sacrum et vertèbres caudales. Concernant le crâne, si certains de ces fragments sont bien documentés (frontal notamment), la sous représentation flagrante des maxillaires et des dents supérieures isolées est surprenante. L'hypothèse d'un apport exclusif des calottes à l'intérieur de la grotte pour en extraire les bois peut être envisagée. La sous représentation de la cage thoracique nécessite quant à elle d'être relativisée du fait de la présence de nombreux fragments de côtes pour lesquels l'espèce n'a pu être déterminée.

En conclusion, l'excellente préservation des surfaces osseuses, la présence de tout petits os (carpiens, sésamoï-

\begin{tabular}{|c|c|c|c|c|c|c|}
\hline \multirow[b]{2}{*}{ Parties squelettiques } & \multicolumn{4}{|c|}{ NMPS } & \multirow[b]{2}{*}{ NMIf/NMIc } & \multirow[b]{2}{*}{$\% \mathrm{PO}$} \\
\hline & $\mathbf{G}$ & D & NL & Total & & \\
\hline Bois & 3 & 4 & & 7 & 2 & 23,3 \\
\hline Crâne & & & 2 & 2 & 2 & 13,3 \\
\hline Dents sup isolées & & & 2 & 2 & 1 & 0 \\
\hline Maxillaire & & & & 0 & 0 & 0 \\
\hline Dents inf isolées & 29 & 22 & & 51 & 6 & 28,3 \\
\hline Mandibule & 1 & 1 & & 2 & 1 & 6,7 \\
\hline Hyoïde & & & & 0 & 0 & 0 \\
\hline Incisives isolées & 3 & 3 & & 6 & 2 & 5,0 \\
\hline Atlas & & & 2 & 2 & 2 & 13,3 \\
\hline Axis & & & & 0 & 0 & 0,0 \\
\hline Vtb cervicale (3-7) & & & 1 & 1 & 1 & 1,3 \\
\hline Vtb thoracique & & & 2 & 2 & 1 & 1,0 \\
\hline Vtb lombaire & & & & 0 & 0 & 0 \\
\hline Sacrum & & & & 0 & 0 & 0 \\
\hline Vtb caudale & & & & 0 & 0 & 0 \\
\hline Côte & & & & 2 & 1 & 0,5 \\
\hline Sternèbre & & & & 0 & 0 & 0 \\
\hline Scapula & 2 & 1 & & 3 & 2 & 10 \\
\hline Humerus & 3 & 5 & & 8 & 5 & 26,7 \\
\hline Radio-ulna & 4 & 2 & & 6 & 4 & 20 \\
\hline Total carpe & 11 & 2 & 0 & 13 & 5 & 7,2 \\
\hline $\begin{array}{l}\text { Métacarpes } \\
\text { principaux }\end{array}$ & 13 & 5 & & 18 & 13 & 60 \\
\hline Métacarpes vestigiels & & & 5 & 5 & 3 & 8,3 \\
\hline Os coxaux & 1 & 1 & & 2 & 1 & 6,7 \\
\hline Fémur & & & 4 & 4 & 2 & 13,3 \\
\hline Patella & 2 & 1 & & 3 & 2 & 10 \\
\hline Tibia & 2 & 2 & & 4 & 2 & 13,3 \\
\hline Os malléolaire & & & & 0 & 0 & 0 \\
\hline Talus & 1 & 2 & & 3 & 2 & 10 \\
\hline Calcaneus & 6 & 2 & & 8 & 6 & 26,7 \\
\hline Cubo-naviculaire & 2 & 1 & & 3 & 2 & 10 \\
\hline Grand cunéïforme & 1 & & & 1 & 1 & 3,3 \\
\hline Petit cunéïforme & & & & 0 & 0 & 0 \\
\hline Total tarse & 10 & 5 & 0 & 15 & 6 & 10 \\
\hline $\begin{array}{l}\text { Métatarses } \\
\text { principaux }\end{array}$ & 7 & 8 & & 15 & 8 & 50 \\
\hline Phalange 1 & & & 28 & 28 & 4 & 23,3 \\
\hline Phalange 2 & & & 18 & 18 & 3 & 15,0 \\
\hline Phalange 3 & & & 4 & 4 & 1 & 3,3 \\
\hline Phalange vestigielle & & & & 0 & 0 & 0 \\
\hline Grand sésamoïde & & & 3 & 3 & 1 & 1,3 \\
\hline Petit sésamoïde & & & & 0 & 0 & 0 \\
\hline Total & 80 & 60 & 71 & 241 & $15^{*}$ & \\
\hline
\end{tabular}

*Le NMlc de 15 a été calculé par combinaison des âges dentaires et des stades d'épiphysation

$\Delta$ TABlEaU 2. Composition des restes de renne de la grotte Rochefort (NMPS, nombre minimum par partie squelettique; G, gauche; D, droit; NL, non latéralisé; NMIf; nombre minimum d'individu de fréquence; NMIc, nombre minimum d'individu de combinaison; PO, pourcentage par os).

(2) Le \% PO (d'après Grigson et Mellars 1987) se calcule, pour chaque partie anatomique, en considérant le NMPS total divisé par le NMPS attendu pour le NMI le plus élevé de l'espèce, le tout multiplié par cent. Par exemple pour le métacarpe : 5 NMPS droits + 13 NMPS gauche = 18 divisé par 26 (NMPS attendu pour un NMIf de 13), multiplié par $100=69,2 \%$ P0). 


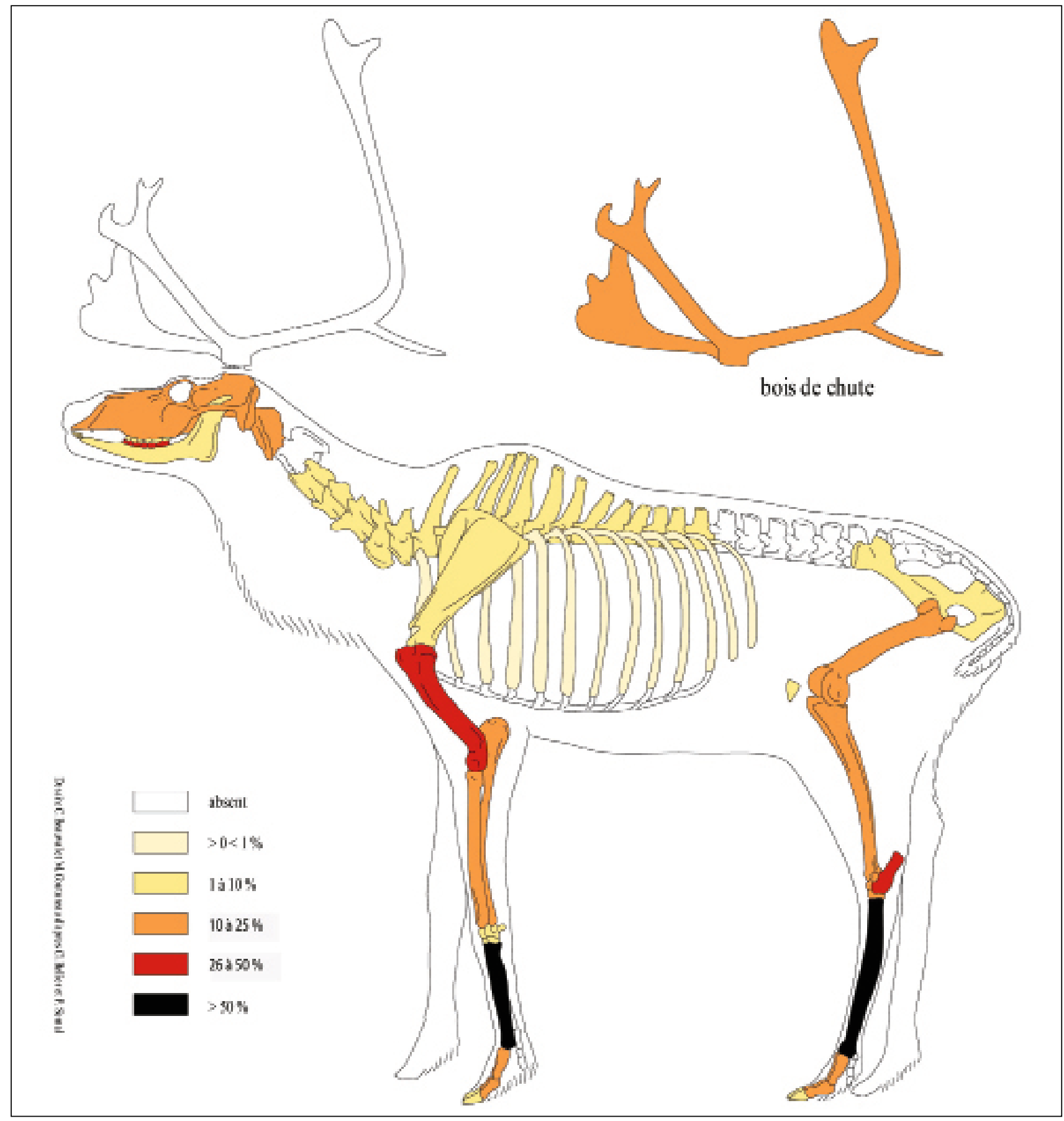

\ Figure 4. Représentation squelettique des restes de renne de la grotte Rochefort $(\odot$ M. Coutureau, htpp/www.archeozoo.org/fr-article50. html et Céline Bemilli, Inrap).

des) ainsi que celle de nombreux fragments de côtes permet d'exclure que la sous représentation des éléments du rachis résulte de phénomènes de conservation différentielle. Les modalités de traitement de la carcasse sont bien plus sûrement responsables de cet état de fait. Lors de la découpe, les solutréens ont sans doute abandonné le rachis ailleurs, sans que la localisation ne soit à ce jour identifiée. Cela peut être sur le site d'abattage et de traitement primaire, sur le devant du porche de la grotte ou encore dans une zone non encore fouillée de celle-ci. L'absence d'axis s'explique peut être par son altération lors de la séparation entre le rachis et la tête.

L'exploitation des rennes par les solutréens se traduit par la présence de traces de découpe dont le nombre demeure toutefois assez restreint $(\mathrm{N}=17)$. Elles documentent essentiellement les phases de désarticulation (Fig. 5, Hinguant et Colleter 2010). Les indices de décharnement sont en effet surtout observables sur des diaphyses indéterminées. Des points d'impact et la présence d'esquilles de percussion indiquent une fracturation volontaire sur place pour extraire la graisse et la 


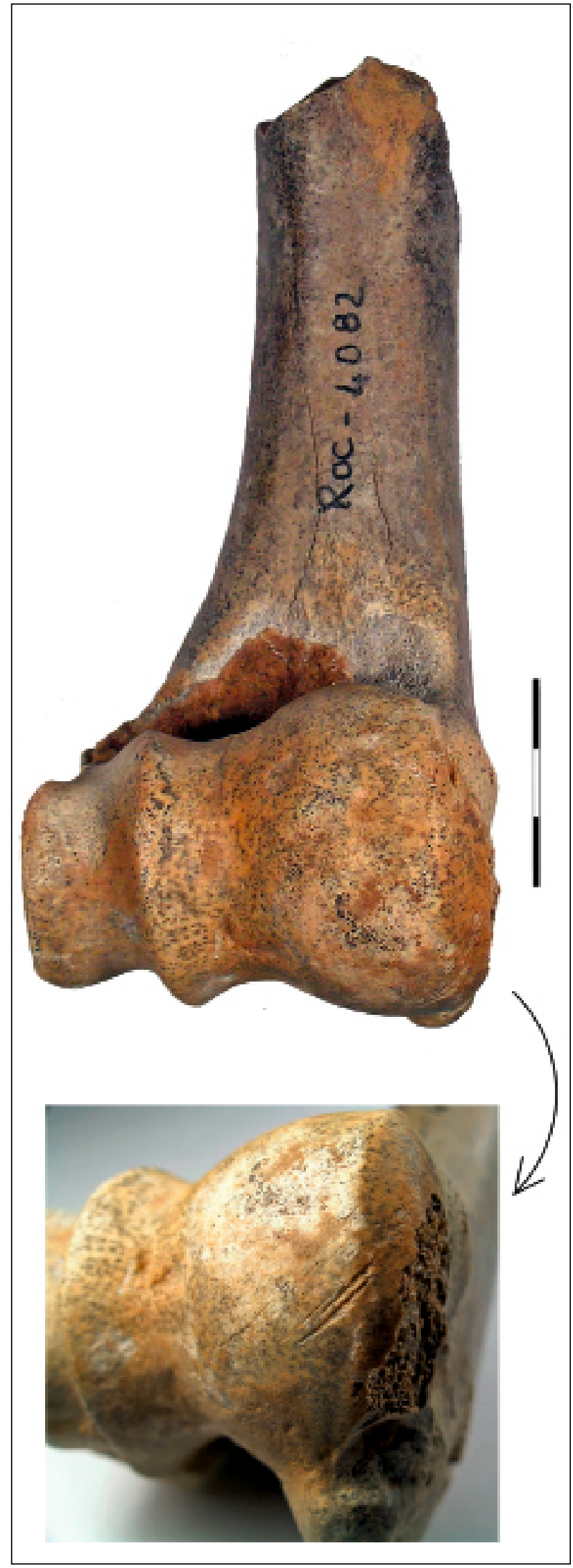

\ Figure 5. Exemple de trace de découpe sur une extrémité distale d'humérus de renne $(\odot$ Pierre-Elie Moullé, Rozenn Colleter, Inrap).

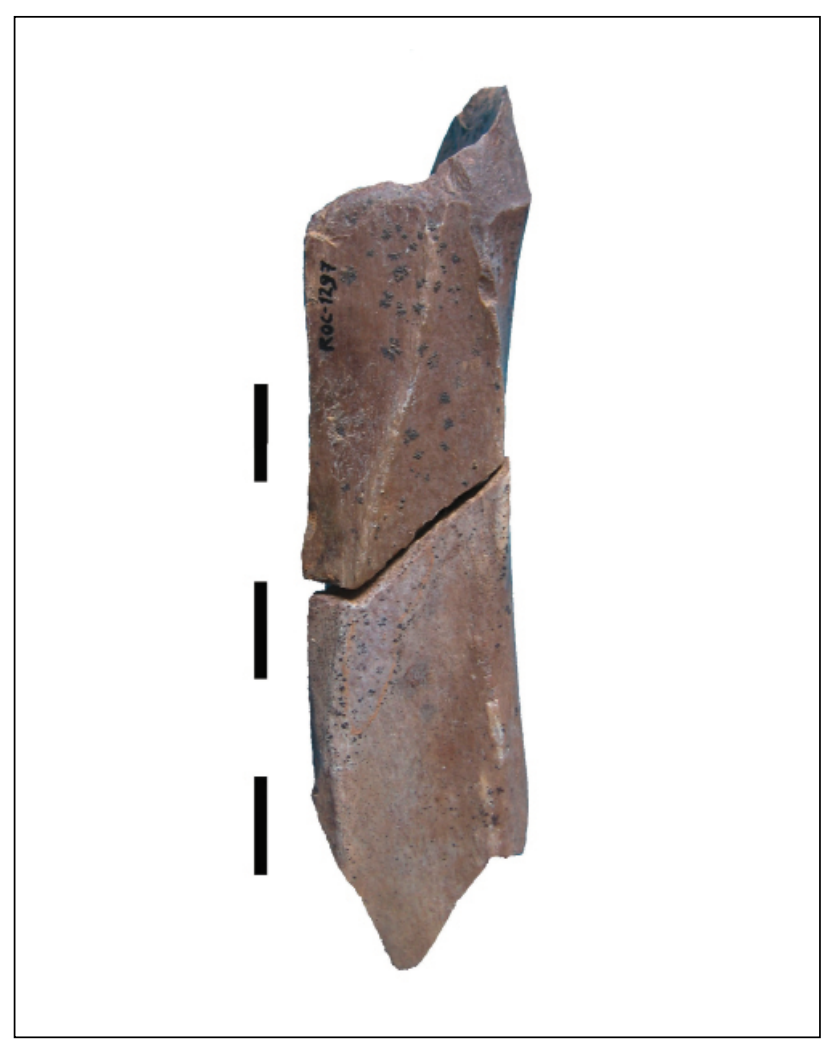

$\Delta$ Figure 6. Exemple de recollage osseux de renne ( $\odot$ Céline Bemilli, Inrap).

moelle. Quelques recollages ont été effectués témoignant de la faible dispersion post-dépositionnelle des vestiges (Fig. 6).

\subsection{Les restes de cheval}

Avec 252 restes déterminés, soit 33,8 \% du NRDt pour un nombre de huit individus, le cheval est la deuxième espèce la plus représentée (Tab. 3).

La répartition anatomique de ces restes se révèle très déséquilibrée entre le squelette céphalique (74 \% NR) -en particulier les dents- et le squelette post-crânien (26 \% NR) (Fig. 7). En fait c'est le nombre de restes dentaires qui est particulièrement élevé avec 169 fossiles (67 \% NR).

Le squelette axial (rachis et cage thoracique) est presque totalement absent, de même que les ceintures. Pour ce qui est de la cage thoracique, il est possible que des fragments de côtes appartenant à ce taxon se trouvent parmi celles dont l'espèce n'a pu être déterminée.

A l'opposé, les éléments les mieux documentés proviennent du squelette céphalique et en particulier des dents isolées (entre 26,2 \% PO pour les incisives et 41,7\% PO pour les jugales inférieures). Le crâne au sens strict est documenté à hauteur de 28,6 \% PO.

Au niveau des membres, les humérus sont absents alors que les fémurs sont très bien figurés et que les radius sont correctement représentés avec $21,4 \%$ PO. Les tibias présentent les mêmes taux de survie que les radius tandis que les 
métapodes atteignent 14,3 \% PO. La représentation des phalanges n'est pas décroissante comme on l'observe souvent puisque les premières affichent un taux de survie de 10,7 \% $\mathrm{PO}$, inférieur à celui des deuxièmes (14,3\% P0). Les troisièmes sont moins bien figurées avec 7,1\% PO. On soulignera l'absence des talus et calcanéum qui sont pourtant les os du tarse les plus compacts tandis que certains carpiens, plus petits, sont documentés.

\begin{tabular}{|c|c|c|c|c|c|c|}
\hline \multirow[b]{2}{*}{ Parties squelettiques } & \multicolumn{4}{|c|}{ NMPS } & \multirow[b]{2}{*}{ NMlc } & \multirow[b]{2}{*}{$\%$ PO } \\
\hline & G & D & NL & Total & & \\
\hline Crâne & & & 2 & 2 & 2 & 28,6 \\
\hline Dents supérieures & & & 28 & 28 & 8 & 33,3 \\
\hline Mandibule & & & & 3 & & 21,4 \\
\hline Dents inférieures & & & 35 & 35 & 8 & 41,7 \\
\hline Canine & & & 2 & 2 & & 14,3 \\
\hline Incisive & & & & 27 & 8 & 64,3 \\
\hline Vertèbre cervicale & & & & 0 & 0 & 0 \\
\hline Vertèbre thoracique & & & & 0 & 0 & 0 \\
\hline Vertèbre lombaire & & & & 1 & 1 & 2,4 \\
\hline Côte & & & & 0 & 0 & 0 \\
\hline Scapula & & & & 0 & 0 & 0 \\
\hline Humerus & & & & 0 & 0 & 0 \\
\hline Radio-ulna & 2 & 1 & & 3 & 2 & 21,4 \\
\hline Pyramidal & & 1 & & 1 & 1 & 7,1 \\
\hline Capitatum & & 1 & & 1 & 1 & 7,1 \\
\hline Lunatum & & 1 & & 1 & 1 & 7,1 \\
\hline Carpien & 1 & & & 1 & 1 & 0 \\
\hline Métacarpien & & 1 & & 1 & 1 & 7,1 \\
\hline Métacarpien II & & & 1 & 1 & 1 & 7,1 \\
\hline Coxal & & & 0 & 0 & 0 & 0 \\
\hline Fémur & 1 & & 3 & 4 & 3 & 28,6 \\
\hline Tibia & 1 & 2 & & 3 & 2 & 21,4 \\
\hline Patella & & 1 & 1 & 2 & 1 & 14,3 \\
\hline Naviculaire & 1 & & & 1 & 1 & 7,1 \\
\hline Grand cunéiforme & 1 & & & 1 & 1 & 7,1 \\
\hline Petit cunéiforme & 1 & & & 1 & 1 & 7,1 \\
\hline Métapode & & & 2 & 2 & 1 & 14,3 \\
\hline Métapode II ou IV & & & 1 & 1 & 1 & 7,1 \\
\hline Grand sésamoïde & & & 1 & 1 & 1 & 7,1 \\
\hline Phalange proximale & & & 3 & 3 & 1 & 10,7 \\
\hline $\begin{array}{l}\text { Phalange } \\
\text { intermédiaire }\end{array}$ & & & 4 & 4 & 1 & 14,3 \\
\hline Phalange distale & & & 2 & 2 & 1 & 7,1 \\
\hline Total & 8 & 8 & 85 & 132 & 8 & \\
\hline
\end{tabular}

A Tableau 3. Composition des restes de cheval de la grotte Rochefort (NMPS, nombre minimum par partie squelettique; G, gauche; D, droit; NL, non latéralisé; NMIc, nombre minimum d'individu de combinaison; $\mathrm{PO}$, pourcentage par os).

Cette représentation des parties squelettiques -même si les effectifs ne sont pas très élevés- est assez instructive sur les étapes du traitement des carcasses de chevaux par les solutréens de la vallée de l'Erve.

Certaines observations faites sur le renne sont valables également pour le cheval, à savoir que les sous représenta- tions de parties squelettiques observées ne sont pas imputables à des phénomènes de conservation différentielle mais bien aux modalités de traitements des carcasses.

L'abandon du rachis à l'extérieur de la grotte est encore plus probable pour les chevaux que pour les rennes vue la masse qu'ils représentent. Pourtant, on remarque que les crânes ont été introduits dans la grotte. On peut envisager que l'objectif était d'extraire la langue et la cervelle, comme en témoigne le recollage de trois fragments de crâne intentionnellement fracturés. De même les membres ont été apporté entiers sans élimination préalable des extrémités (acropodes).

L'exploitation des chevaux par les solutréens se traduit par la présence de traces de découpe sur 17 restes $(4,5 \%)$ qui documentent les phases de dépouillage, de désarticulation, mais surtout de prélèvement de la viande (Fig. 8). Les quelques traces d'impact et des fractures systématiques en spirale sur les os longs témoignent, quant à elles, de l'extraction de la moelle et de la graisse (Fig. 9). Enfin, les quelques stigmates de brûlure peuvent, en revanche, refléter soit un mode de cuisson soit le rejet des déchets dans des zones de combustion.

\section{SAISONS D’ABATTAGE}

L'analyse des âges (cf. supra) permet d'avancer quelques données concernant la saisonnalité d'occupation de la grotte par les solutréens car des individus juvéniles sont présents pour les deux espèces. Les âges d'abattage des faons indiquent une occupation entre l'été et l'automne tandis que ceux des poulains la situe plutôt au printemps (Fig.10). Ceci implique que les chasseurs ont occupés la grotte durant une à deux saisons consécutives ou bien qu'ils y sont revenus régulièrement durant ces deux saisons.

\section{INDUSTRIE OSSEUSE}

Lors de l'analyse archéozoologique, une trentaine de pièces a été isolée car présentant des stigmates de transformation et/ou d'utilisation. II s'agit principalement d'os à impressions appelés le plus souvent retouchoir. L'observation minutieuse de ces pièces nous a amené à plutôt envisager leur utilisation comme des presseurs/compresseurs. L'une d'entre-elle (Fig. 11) présente une usure par écrasement et un micro esquillement, associé à une esquille plus importante, longue et rasante, qui semble procéder de l'utilisation de la pièce par percussion posée.

L'hypothèse concernant la fonction de cette pièce comme un presseur pour retoucher les pièces lithiques foliacées a motivée une expérimentation réalisée par Miguel 


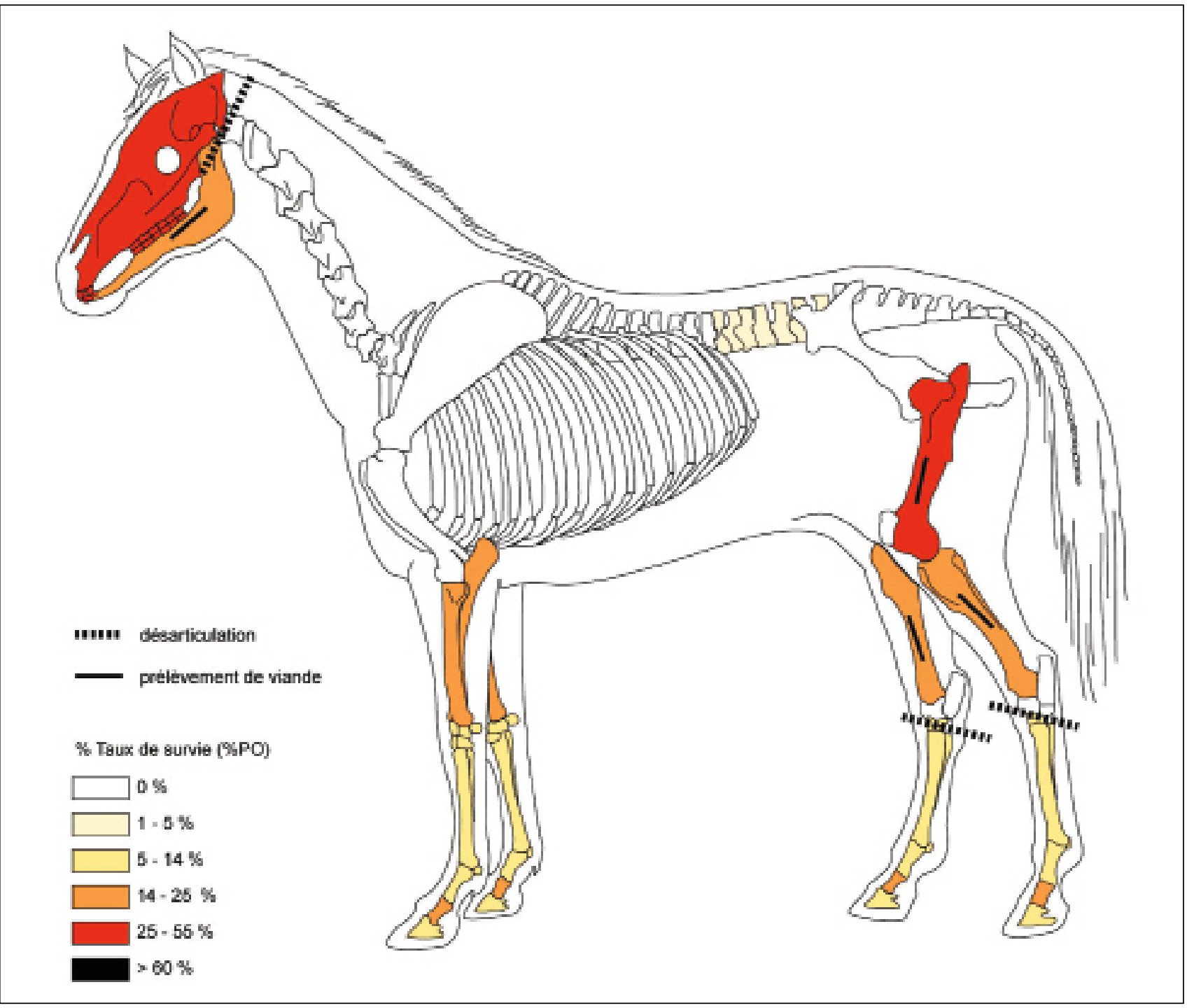

\ FiguRE 7. Représentation squelettique des restes de cheval de la grotte Rochefort $\left({ }^{\circ} \mathrm{M}\right.$. Coutureau, htpp/www.archeozoo.org/fr-article50. html et Céline Bemilli, Inrap).

Biard et les résultats obtenus expérimentalement ont reproduit exactement les observations faites sur la pièce archéologique.

\section{CONCLUSION}

Au sein des restes osseux fauniques conservés dans la couche solutréenne de la grotte Rochefort, traduisant un spectre particulièrement étoffé, deux grands herbivores prédominent donc dans les choix cynégétiques des chasseurs; le renne et le cheval.

La préservation exceptionnelle des restes osseux dans ce site permet une analyse vraiment complète des modalités d'acquisition et d'exploitation du gibier par les solutréens. L'ensemble faunique étudié correspond à ce jour aux seuls vestiges découverts dans une zone périphérique de l'occupation, expliquant peut être les profils squelettiques observés. Ainsi, les premières phases de traitement (dépouillage et découpe en segments) ne sont a priori pas documentées et ont pu avoir lieu à l'extérieur de la grotte ou encore sur le site d'abattage. Le faible nombre de traces de découpe observées sur les os reflète pour sa part une parfaite maîtrise des gestes et sans doute l'utilisation de lames ou d'éclats bruts.

Quoi qu'il en soit les résultats traduisent une exploitation intense des carcasses pour les deux principales espèces. Les phases de désarticulation et de décharnement ont été effectuées à l'intérieur de la grotte et les os ont ensuite été systématiquement fracturés pour en récupérer la moelle et la graisse. Les fragments osseux ainsi produits ont ensuite servi, sans doute de manière opportuniste, pour des activités techniques non alimentaires.

Si les résultats sur la saisonnalité reposent sur peu d'individus, ils attestent avec certitude d'au moins plusieurs séjours, peut-être distincts, dans la grotte : durant l'automne, d'après les estimations d'âge d'abattage 


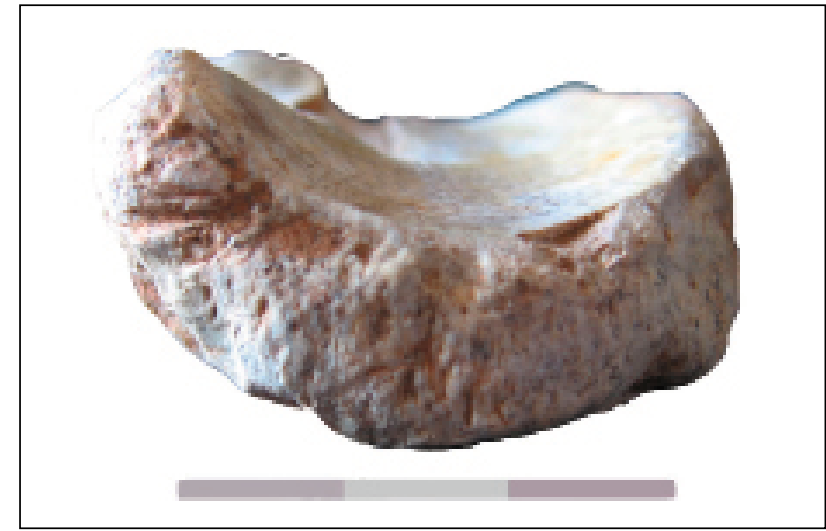

A Figure 8. Exemple de trace de découpe sur un os du carpe de cheval (๑ Céline Bemilli, Inrap).

des rennes, et au printemps, d'après les estimations d'âge d'abattage des chevaux. Le fait qu'un certain nombre de fragments osseux présente des stigmates dus à l'action de petits carnivores confirme l'absence périodique de l'homme dans la cavité. En l'état des données actuelles, le niveau solutréen de la grotte Rochefort apparaît comme une succession d'occupation périodique de moyenne durée très rapprochées dans le temps. Les vestiges fauniques témoignent de l'exploitation d'un environnement relativement riche que la particularité du canyon de Saulges a peut être privilégié.

\section{REMERCIEMENTS}

Les recherches sont menées dans le cadre du programme "les occupations paléolithiques de la vallée de I'Erve " de I'UMR 6566 du CNRS, "Centre de Recherches en Archéologie, Archéosciences, Histoire ". Elles sont financées par le Ministère de la Culture (DRAC, Service régional de

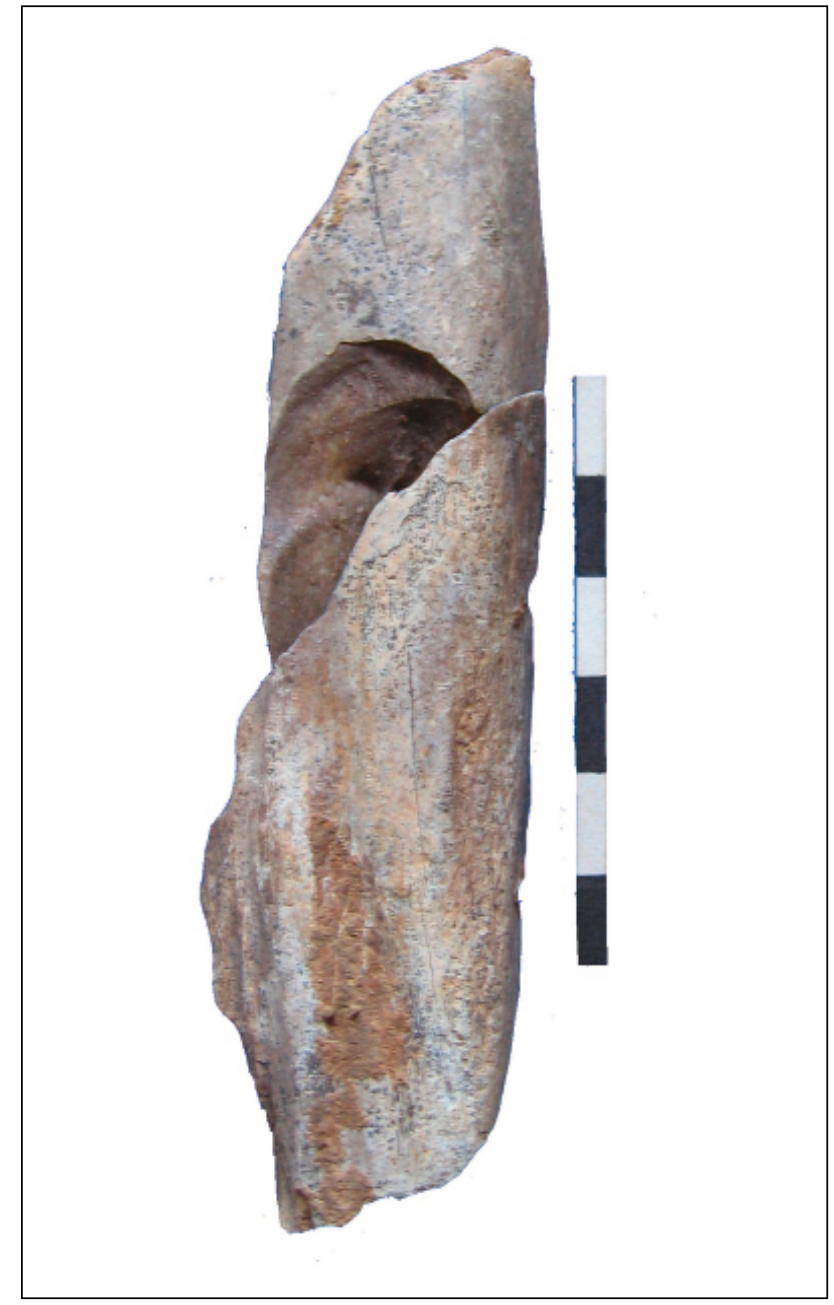

$\Delta$ Figure 9. Exemple de recollage osseux de cheval (๑ Céline Bemilli, Inrap).

l'archéologie des Pays de la Loire), le Conseil général de la Mayenne et l'Institut national de recherches archéologiques préventives (INRAP). •

\section{BIBLIOGRAPHIE}

BINFORD, L.R. 1981: Bones. Ancient man and modern myths. Academic Press. New York, San Francisco, London.

Bouchud, J. 1966: Essai sur le renne et la climatologie du Paléolithique moyen et supérieur. Imprimerie Magne. Périgueux.

CoRnEVIn, C. et LESBRE, X. 1894: Traité de l'âge des animaux domestiques d'après les dents et les productions épidermiques. Librairie J.-B. BaiIlière et fils. Paris.

DELPECH, F. 2012: "Biostratigraphie des niveaux solutréen de LaugerieHaute (Les Eyzies, Dordogne, France). Implications archéologiques". Paléo 23: 105-116.

GuAdELLI, J.-L. 1998: "Détermination de l'âge des chevaux fossiles et établissement des classes d'âge". Paléo 10: 87-93.

CASTel, J.-C. et MadelaInE, S. 2006: "Quelques éléments remarquables de la faune du Solutréen de Laugerie-Haute (Les-Eyzies-de-Tayac, Dordogne)". Paléo 18: 275-284.
GRAYSON, D. K. 1984: Quantitative zooarchaeology: topics in the analysis of archaeological faunas. Studies in Archaeological Science. Academic Press. Orlando.

Grigson, C. et MelLaRS, P. 1987: "The mammalian remains from the middens'". In P. Mellars (ed.): Excavations on Oronsay. Prehistoric Human Ecology on a Small Island. Edinburgh University Press. Edinburgh: 243-289.

HINGUANT, S. et BIARD, M. 2013: "Le Paléolithique supérieur ancien de la vallée de l'Erve (Mayenne): un état des connaissances". In P. Bodu, L. Chehmana, L. Klaric, L. Mevel, S. Soriano et N. Teyssandier (dir.): Le Paléolithique supérieur ancien de l'Europe du Nord-Ouest : réflexions et synthèses à partir d'un projet collectif de recherche sur le centre et le sud du Bassin parisien. Actes du colloque européen "Le Paléolithique supérieur ancien de l'Europe du Nord-Ouest (3500015000 BP)", séance de la Société préhistorique française, Sens, 15-18 avril 2009. Société préhistorique française. Mémoire 56. Paris: 239-250. 


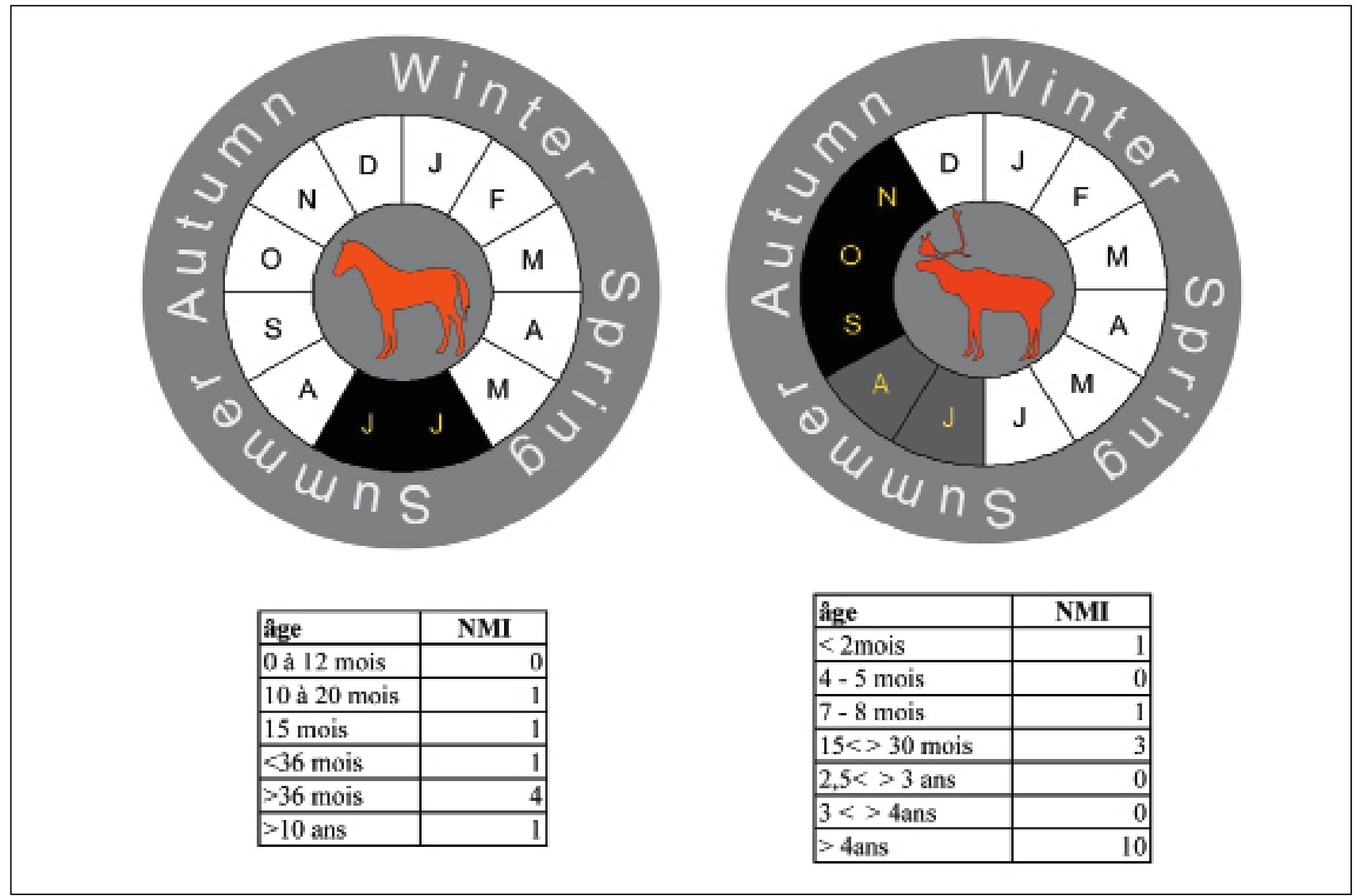

\ FiguRE 10. Grotte Rochefort, âges et saisons d'abattage des chevaux et des rennes ( $\odot$ Céline Bemilli, Inrap).

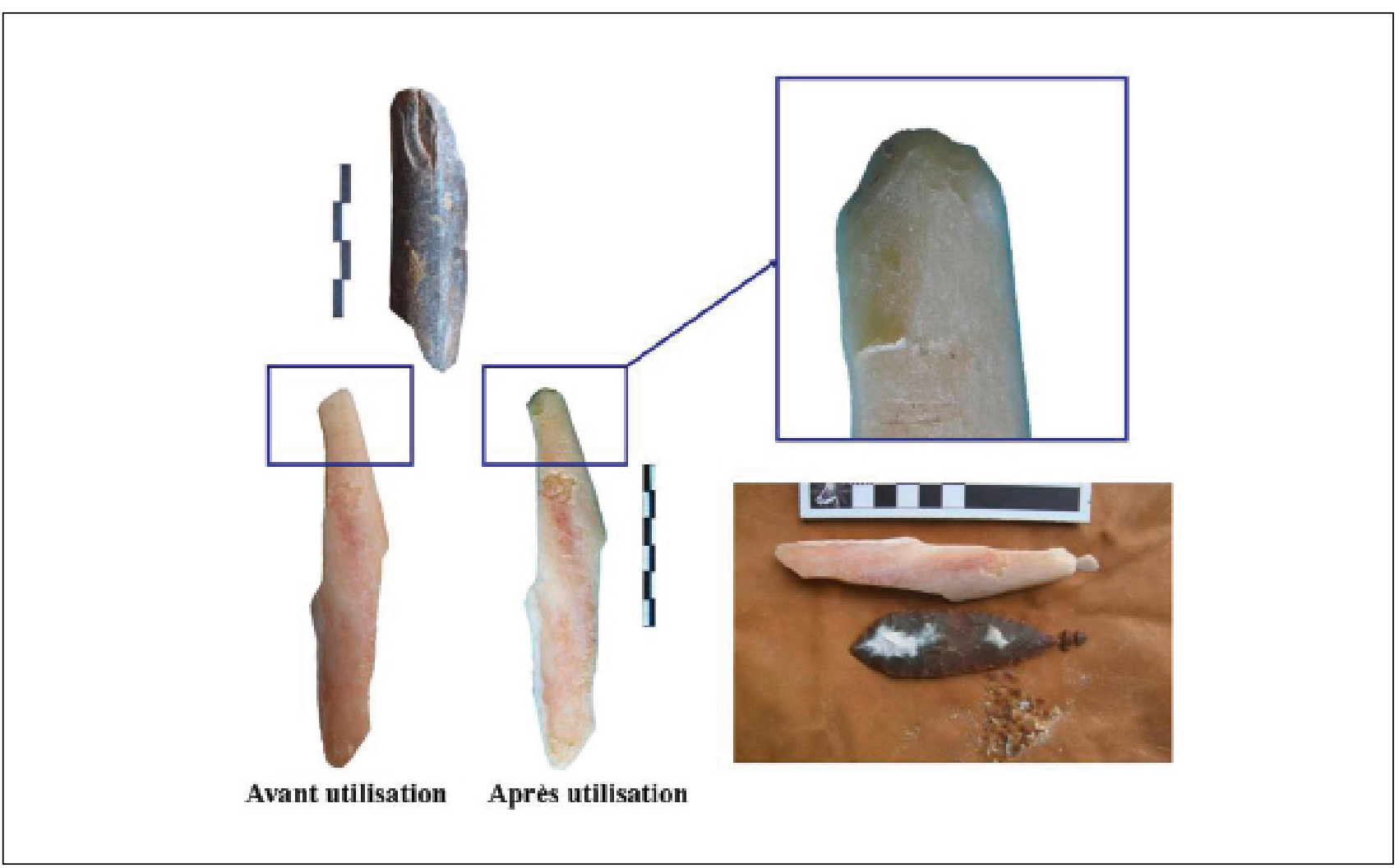

\ FIGURE 11. Retouchoir/presseur archéologique de la grotte Rochefort et pièce expérimentale réalisée par M. Biard ( $\odot$ Céline Bemilli et Stéphan Hinguant, Inrap). 
Hinguant, S., Moullé, P.-É., Arellano, A. et Pigeaud, R. 2012: "Pièces osseuses gravées du Solutréen moyen de la grotte Rochefort (SaintPierre-sur-Erve, Mayenne)". Paléo 12: 337-356.

Hinguant, S. et Colleter, R. (dir.) avec les contributions de Arellano, A. Béarez, P., Bemilli, C., Biard, M., Bodinier, B., Chaut, J.-J., Debret, M., Desclaux, E., El Guennouni, K., Hanquet, C., Laignel, B., Lenormand, A., Marcoux, N., Moullé, P.-É., Noël, F., Peschaux, C., Pigeaud, R., PomMier, V., Rodet, J., Roger, T., TSObgou Ahoupé, R. et VISSAC, C. 2010: Le Solutréen de la grotte Rochefort (Saint-Pierre-sur-Erve, Mayenne). Rapport de fin d'opération triennale 2008-2010, campagne 2010 (3/3). Service régional de l'archéologie des Pays de la Loire. Rennes et Nantes.

HufthAmmer, A. K. 1995: "Age determination of Reindeer (Rangifer tarandus L.)". Archaeozoologia VII/2: 33-41.
MAdelaINE, S. 1989: "Contribution des anciennes fouilles à la connaissance des ongulés et de leur milieu durant le Würm récent en Dordogne". Paléo 1: 36-46.

PopLIN, F. 1976a: "Remarques théoriques et pratiques sur les unités utilisées dans les études d'ostéologie quantitative, particulièrement en archéologie préhistorique". J. Combier (dir.): Thèmes spécialisés. $B$. Problèmes ethnographiques des vestiges osseux, actes du IXe Congrès de l'UISPP, Nice, 1976. Paris: 125-141.

- 1976b: "A propos du nombre de restes et du nombre d'individus dans les échantillons d'ossements". Cahier du Centre de Recherche Préhistorique 5: 61-76.

- 1976c: Les grands vertébrés de Gönnersdorf, fouille 1968. Franz Steiner, Verlag GMBH. Wiesbaden.

SPIESS, A. E. 1979: Reindeer and Caribou hunters: An Archaeological Study. Academic Press. New York. 
